\title{
Control methods for the coordination of autonomous vehicles at intersections
}

\author{
Zsófia Farkas and András Mihály and Péter Gáspár
}

\begin{abstract}
The paper defines the main challenges related to the determination of the ordering of vehicles at an intersection and proposes a general coordination method for autonomous vehicles to avoid collisions in intersection scenarios. The minimization of traveling time and energy optimality as performances of vehicles are incorporated in the iterative control procedure, and the goal of the research is to secure the collision-free passage of autonomous vehicles through the intersection. The proposed coordination method is demonstrated by several simulation examples made in a high-accuracy simulation environment to present its efficiency.
\end{abstract}

\section{INTRODUCTION}

The control of vehicles at intersections signifies important challenges in traffic systems. The safe passage of the vehicles at the intersection is ensured by the corresponding traffic rules and their adherence. These rules can be considered as the basic condition for the use of autonomous vehicles at the traffic system. Therefore flexible solutions are needed to ensure the collision-free crossing of the intersection taking into consideration the minimization of time as a probable purpose of the control design. The minimum traveling time, as the performance of the vehicles effects on the determination of the ordering of vehicles passing through the intersection.

As the coordination of vehicles at the intersection has become an important academic research topic, several papers have proposed various strategies for connected or autonomous and human-driven vehicles. A comprehensive survey about non-signalized and signalized intersections is made by [1]. Plenty of solutions are presented in that paper focused on time slots, trajectory planning, virtual traffic lights. A multi-agent system developed for the control of intersections without traffic lights and signs is approached in several works like [2]. Another multi-agent system in which autonomous agents are the driverless vehicles at the non-signalized intersection is introduced by [3]. The proposed heuristic optimization algorithm is used to prevent the collision of vehicles and decrease time delay at the intersection. For the purpose of collision avoidance of autonomous vehicles at intersections, the optimization problem is reformulated as a convex program and solved by [4]. An intersection control

F. Zsófia is with Department of Control for Transportation and Vehicle Systems, Budapest University of Technology and Economics, Stoczek u. 2, H-1111 Budapest, Hungary. E-mail: farkas.zsofia@mail.bme.hu

A. Mihály and P. Gáspár are with Systems and Control Laboratory, Institute for Computer Science and Control, Kende u. 13-17, H-1111, Budapest, Hungary. E-mail: [andras.mihaly;peter.gaspar]@sztaki.hu

The research was supported by the Hungarian Government and cofinanced by the European Social Fund through the project "Talent management in autonomous vehicle control technologies" (EFOP-3.6.3-VEKOP16-2017-00001) model is introduced not only to guarantee collision avoidance but the travel experience, see [5]. The intersection is divided into collision areas and a schedule rule is designed to determine the priorities of the vehicles. An intersection crossing strategy by using a centralized controller is proposed by [6], while a decentralized solution solving the local optimization problems of the sequence of the vehicles is developed by [7]. A bilevel, model predictive controller with coordination and vehicle levels is presented by [8] to ensure the collision avoidance between automated vehicles at intersections. The optimization problem is solved by the use of a distributed Sequential Quadratic Programming method. A model for the intersection problem considering the acceleration and time constraints and by using a car-following model is proposed by [9]. The developed solution is demonstrated by several simulations and compared to other operations of intersections as the use of traffic lights or roundabouts. Two speed guidance algorithms for single and multiple vehicles to achieve the minimum traveling time at the intersection are proposed by [10]. A coordination method, taking into consideration the most important performances like traveling time and energy consumption is introduced in [11]. The proposed strategy is modeled by an intersection scenario with three vehicles having different characteristics. A cooperative vehicle intersection control algorithm is proposed to affect the behavior of the vehicles to ensure safe crossing of the intersection, see [12]. An optimal scheduling of autonomous vehicles arriving at the intersection considering the ordering problem as a Mixed-Integer Linear Program is proposed by [13].

Although several solutions are proposed for the control design of vehicles in intersection scenarios, the contribution of the paper is a control method for autonomous vehicles crossing the intersection without collision at the minimum time or energy consumption. The coordination method is based on the performances of the autonomous vehicles like the minimum of their traveling time during the passage through the intersection and the minimum fuel consumption, see [11], [14]. Speed limits along with maximum and minimum accelerations are taken into consideration in the control solution which is suitable for the determination of the ordering of autonomous vehicles at intersections.

The paper is organized as follows. The coordination challenge of autonomous vehicles at the intersection is defined in Section II. Section III describes the components of the control design, introduces the intersection scenario, contains dynamical constraints and the determination of traveling time as well as the control procedure. The method is presented 
through simulation examples in Section IV. Conclusion remarks are presented in Section V.

\section{Problem statement}

The base for the research is to determine the control design challenges related to the coordination of autonomous vehicles at intersection scenarios. Traditionally the coordination of traffic at intersections is solved by traffic signs and lights, and their adherence is dependent upon the driver behavior, therefore the exact, determinate ordering of vehicles is given. In case of the development of intelligent traffic system, infrastructure and with the appearance of highly automated vehicles, the determination of the passing order through the intersections and the design of control methods for the autonomous vehicles have become a difficult challenge. The coordination of autonomous vehicles at an intersection can be more complex by

- the increasing number of the vehicles,

- the change of their driving directions,

- the passage of vehicles with priority,

- the vehicles having characteristics related to danger,

- the defined purpose of the control design of the vehicles like the minimization of time, energy losses and fuel consumption.

In case of a non-signalized intersection the opportunity of collision is arisen in a traffic situation consisted of two autonomous vehicles crossing their directions. An intersection scenario with several autonomous vehicles, traveling straight and turning left or right, is proved to signify more complex problem in designing control for the crossing of the vehicles, see Figure 1. A control design with multiple steps is needed for the control of autonomous vehicles crossing an intersection in which several vehicles follow the arriving vehicles.

The proposed method considers most of the abovementioned conditions increasing the complexity of the control of the intersection. Speed and acceleration limits, the condition of collision-avoidance are built in the control design. The minimization of traveling time and energy of the vehicles passing through the intersection are in the focus of the coordination method while the oncoming traffic is left out of consideration. Furthermore it is assumed that the distance of the vehicles from the origin of the intersection, their velocities and driving intentions are measured and known for each participants of the traffic and a coordinator by using V2X communication methods.

Related to this field of control design there are several papers focusing on maneuvers and motion prediction of autonomous vehicles, see [15], [16], [17]. In connection with the driving intention of autonomous vehicles, other works approaches the detection problem of the maneuvers of vehicles considering the alert signals, like brake lights and turn signals, see [18], [19]. A particular area of interest is the communication methods between the participants of the traffic and the infrastructures [20], [21].

\section{INTERSECTION CONTROL DESIGN}

\section{A. Intersection scenario}

In the proposed intersection scenario vehicles can drive straight, turn left or right, therefore there is the opportunity of collisions between vehicles tracking the same or different trajectories, [22]. The goal of the paper is to design an algorithm which guarantees collision-free passage of autonomous vehicles at intersections, while it minimizes the total traveling time or the energy consumed by the vehicles. Therefore, congestion at the intersection can be avoided and fuel consumption can be reduced while safety of the autonomous vehicles can be enhanced. In view of the intersection scenario, the goal of the centralized controller is to determine the ordering of autonomous vehicles as well as their accelerations with the consideration of total traveling time or energy consumption, and to guarantee collisionavoidance at the same time.

The proposed analytical control algorithm is based on an iterative calculation of accelerations of the autonomous vehicle, with some pre-assumptions. The intersection is considered a four-directional double lane intersection, divided into several zones depicted in Figure 1.

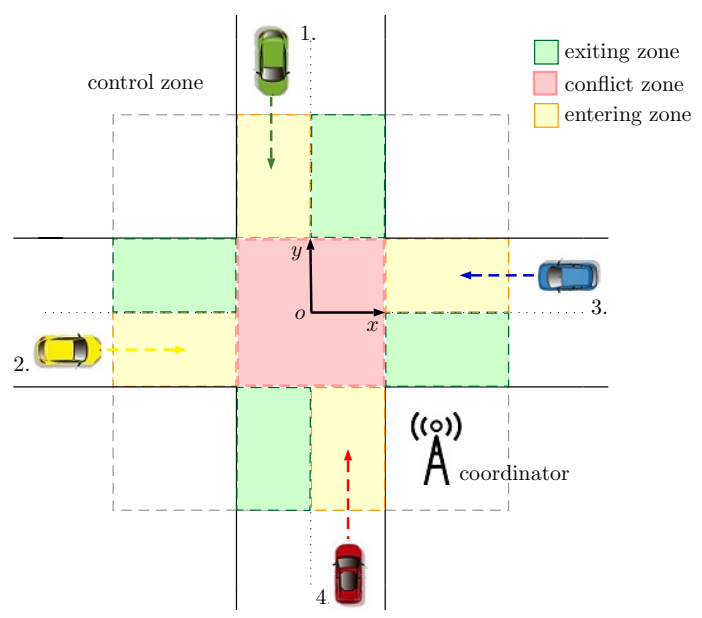

Fig. 1. Illustration of intersection scenario with the relevant zones for the control design

Outside the control zone, the autonomous vehicles are controlled individually. As they reach the control zone, the centralized controller calculates a velocity profile for each vehicle, based on their turning intentions and actual velocities, and sends the necessary accelerations for the vehicles using $V 2 I$ communication. Although the iterative calculation considers maximum four vehicles at the intersection at the same time, the calculation is repeated with reconfigured initial conditions determined for the vehicles when a new vehicle enters the intersection.

\section{B. Conditions for the control design}

In case of autonomous vehicles it is also necessary to give speed limits to secure safety during the passage of the vehicles through the intersection. The safe velocities for 
the autonomous vehicles are defined regarding their turning intentions. Thus, a velocity for left turn $v_{l}=\sqrt{R_{l} g \mu}$ and right turn $v_{r} \sqrt{R_{r} g \mu}$ and for heading straight $v_{s}=v_{l i m}$ are calculated using simplified vehicle dynamics, where $R_{l}$ and $R_{r}$ are the turning radius of the left and right-hand turn at the intersection, $g$ is the gravitational constant, $\mu$ is the tireroad adhesion coefficient assumed to be known by estimation methods, see e.g. [23], [24], [25], $v_{\text {lim }}$ is the regulated speed limit at the intersection. In a typical intersection scenario with four directions $n=4$ and a lane width of 5 meters, considering $\mu=0.8$ tire-road adhesion coefficient, the safe velocity for turning right is approximately $v_{r} \approx 16 \mathrm{~km} / \mathrm{h}$, for turning left $v_{l} \approx 28 \mathrm{~km} / \mathrm{h}$, while the speed limit is $v_{\text {lim }}=50 \mathrm{~km} / \mathrm{h}$. Maximum and minimum accelerations are also defined for the autonomous vehicles to ensure passenger comfort, preserve adhesion and prevent skidding of the wheels. In the paper, threshold values of $a_{\max }=5$ $\mathrm{m} / \mathrm{s}^{2}$ and $a_{\min }=-5 \mathrm{~m} / \mathrm{s}^{2}$ are selected, see [26].

For collision-avoidance some constraints must be built in the control design. The base for the collision-free passage of the autonomous vehicles at the intersection is to set the condition that only one vehicle can be located at the conflict zone of the intersection at the same time. Consequently, the oncoming vehicle (independently of its trajectory) can enter the conflict zone of the intersection only at the exit time of the previous vehicle.

\section{Time-optimal intersection control design}

One of the control objective is to minimize the total traveling time $T_{\text {total }}$ of the autonomous vehicles, consequently to reduce the possibility of congestion emerging at the intersection. This goal is met by ordering and accelerating the vehicles to pass through the intersection with the possible biggest velocities.

Therefore, the control algorithm calculates constant accelerations $a_{i}$ for the entering zone for the vehicles ( $i$ $\in[1 \ldots n])$ by which the maximal velocities for the given trajectory $v_{i, \max }$ can be reached at the end of the entering zone. Accordingly the initial accelerations calculated for the autonomous vehicles are in coherence with the goal to minimize the traveling time for the vehicles. In the first step of the proposed optimization method, the following calculation is processed for all vehicles entering the intersection:

$$
a_{i}=\frac{v_{i, \max ^{2}}-v_{i, 0}^{2}}{2 s_{i, e n t}}
$$

where $v_{i, 0}$ are the initial velocities, $s_{i, \text { ent }}$ are the initial distances from the origin of the intersection for all vehicles $(i \in[1 \ldots n])$.

Corresponding to the conditions given for the accelerations of the vehicles, $a_{i}=\left\{a_{\max } ; a_{\min }\right\}$ are substituted for the calculated accelerations in (1) if the threshold values are reached. In this case, the maximal entering velocity in the conflict zone must be modified as

$$
v_{i, \max }=\sqrt{v_{i, 0^{2}}+2 a_{i} s_{i, \text { ent }}} .
$$

The core of the coordination method is the comparison of the traveling time of the autonomous vehicles at the intersection to prevent collisions during their passage. In the minimization process of the traveling time, the conflict situations (probability of collision) between autonomous vehicles are analyzed to control the accelerations, as the speed of the vehicles to cross the intersection safely. Therefore the modification of the acceleration tracked by the autonomous vehicles before their arrival at the intersection conflict zone is incorporated in the proposed algorithm to avoid collision. When the autonomous vehicles get into a conflict situation, the priority for the passage at the intersection is defined for the vehicle with the smallest traveling time passing through the intersection, i.e. the smallest time means the time when the vehicle leaves the conflict zone. The other vehicles reduce their acceleration in order to give priority for the vehicle leaving firstly the intersection, then give priority for the secondly leaving vehicle in the same manner. In case of multiple vehicles conflict, the algorithm also considers the stopping of vehicles at the end of the entering zone, and defines a waiting time for entering the conflict zone with a predefined acceleration.

For the proposed algorithm, it is necessary to calculate the entrance and exit time of each vehicles for the conflict zone. The time of entrance for vehicles at the intersection can be calculated by solving the following second order equation:

$$
\frac{1}{2} a_{i, 0} t_{i, e n t}^{2}+v_{i, 0} t_{i, e n t}-s_{i, e n t}=0
$$

where $t_{i, e n t} \geq 0 i \in[1 \ldots n]$ is the time of the entrance for all vehicles entering the intersection. By the defined constant accelerations for the entering zone, (3) can be simplified as:

$$
t_{i, \text { ent }}=\frac{s_{i, \text { ent }}}{\left(v_{i, \max }+v_{i, 0}\right) / 2}
$$

The time spent at the intersection by the vehicles is defined as assuming the vehicle to travel with constant speed when it reaches the conflict zone. Therefore the condition $a_{i}=0$ is considered in the conflict zone, thus

$$
t_{i, \mathrm{con}}=s_{i, \text { int }} / v_{i, \max },
$$

where $s_{i, i n t}$ is the length of the trajectory in the conflict zone, depending on the turning intention of the vehicle.

If the situation exists that the autonomous vehicle must stop for giving priority to the other vehicle (or vehicles), a waiting time $t_{i, \text { wait }}$ is calculated. In this case $v_{i, \max }=0$ is set in the calculation, in order to stop the vehicle before the conflict zone. The waiting time is calculated as the difference between final time of the prior vehicle (or vehicles) and entering time $t_{i, e n t}$ of the subject vehicle calculated by (4). After the conflict zone is free, i.e $t>t_{i, e n t}+t_{i \text {,wait }}$, the autonomous vehicle enters the conflict zone with the predefined acceleration $a_{\max }$, then the time spent in the conflict zone is

$$
t_{i, \text { con }}=\sqrt{2 s_{i, i n t} / a_{\max }}
$$


Therefore, the traveling time of the autonomous vehicles at the intersection is the sum of the entrance and exit time:

$$
t_{i, f i n}=t_{i, e n t}+t_{i, w a i t}+t_{i, c o n}
$$

Then the total traveling time is selected as the maximal value of each traveling time, $T_{\text {total }}=\max \left(t_{i, f i n}\right) \quad \forall i \in[1 \ldots \mathrm{n}]$.

Based on the position and velocity measurements sent by the autonomous vehicles to the intersection coordinator, the calculation is evaluated in the following iterative manner:

- Firstly, maximal velocity $v_{i, \max } i \in[1 \ldots n]$ of each vehicles is calculated based on the turning intentions of the vehicles entering the control zone, by which their passage through the intersection conflict zone can be completed. Given the initial velocities and distances from the intersection origin, a constant acceleration $a_{i} i$ $\in[1 \ldots n]$ is calculated for each vehicle using (1). Moreover, if the maximal velocity cannot be reached by the predefined acceleration threshold values, the maximal velocity is modified by substituting $a_{i}=\left(a_{\max } ; a_{\min }\right)$ for the acceleration.

- The entrance time $t_{i, \text { ent }}$ and the exit time $t_{i, f i n} \in[1 \ldots n]$ for the conflict zone is calculated for each vehicle. Then, the possible conflicts are defined by analyzing the overlaps in time spent in the conflict zone by each vehicles. If there is no detected conflict, the autonomous vehicles use the previously calculated accelerations to pass through the intersection.

- In case of a conflict detected between two or more vehicles, the algorithm works as follows: the vehicle having the minimal exit time $t_{i, \text { fin }} i \in[1 \ldots n]$ gets priority, and does not change its previously calculated acceleration. The calculation follows by reducing iteratively the acceleration $a_{i}$ of other vehicles and recalculating their associated entering time $t_{i, e n t}$ by using (4) until their entrance time is bigger than the exit time of the prior vehicle. This iterative calculation process is followed for all the conflicting vehicles. Finally, the resulting accelerations $a_{i} i \in[1 \ldots n]$ are applied for the vehicles to follow in the entering zone. If the waiting time $t_{i, \text { wait }}$ is bigger then zero, the subject vehicle waits for the calculated amount of time at the end of the entering zone (beginning of the conflict zone) and then accelerates with $a_{i}=a_{\max }$.

- Finally, if a new vehicle enters the intersection, the algorithm sets vehicle following mode for it until the previous vehicle leaves the intersection conflict zone. If that happens, the above-mentioned process is repeated with the new initial conditions for all vehicles in the intersection control zone.

\section{Energy-optimal intersection control design}

Another goal of the control design can be the minimization of the total energy consumption of the vehicles entering the intersection. In this design process, the conditions including collision avoidance and dynamical constraints listed in Section III-B are also considered. However, the energy optimality is the key design factor, along with the design of velocity trajectory, in the ordering of the vehicles. Therefore, the main difference from the time-optimal algorithm (detailed in Section III-C) is that the ordering of the vehicles at the intersection is not based on their traveling time, rather their kinetic energy $E_{i, k i n}=0.5 m_{i} v_{i}^{2} i \in[1 \ldots n]$. For the sake of this calculation, it is assumed that each autonomous vehicle sends information about its mass $m_{i} i \in[1 \ldots n]$ for the coordinator besides its initial position and velocity.

The iterative calculation of the vehicle ordering and the velocity design calculation evaluated by the intersection coordinator are the following:

- Maximal velocities $v_{i, \max } i \in[1 \ldots n]$ are calculated for the autonomous vehicles. If the initial velocity $v_{i, 0} i \in$ $[1 \ldots n]$ of the vehicle entering the control zone exceeds its maximal velocity, a constant acceleration $a_{i} i \in$ $[1 \ldots n]$ is calculated for it, by which its velocity can be reduced to the maximal velocity when it enters the conflict zone. Otherwise, $a_{i}=0$ is selected for further calculation.

- Similarly to the time-optimal solution, entrance time $t_{i, e n t}$ and exit time $t_{i, f i n} \in[1 \ldots n]$ are calculated for each vehicle. In case of no conflict detected, the autonomous vehicles use the previously calculated accelerations to pass through the intersection.

- In case of a conflict situation between two or more vehicles, the energy-optimal algorithm works as follows: the vehicle having the maximal kinetic energy $\max \left(E_{i, k i n}\right)$ $i \in[1 \ldots n]$ at the entrance of the conflict zone gets priority, and does not change its previously calculated acceleration. The calculation follows by iteratively reducing the acceleration $a_{i}$ of other vehicles in the same manner as detailed in the time-optimal solution, with the difference in selecting priority based on the kinetic energy. Furthermore the newly entered vehicles are also incorporated in the design.

\section{E. Vehicle control model}

The autonomous vehicle dynamics is controlled by applying a sufficient longitudinal control force coherent with the necessary acceleration $a_{i} i \in[1 \ldots n]$. Therefore, the longitudinal forces are $F_{i, l}=m_{i} a_{i}+F_{i, d}$, where $m_{i} i \in$ [1...n] is the mass of the vehicle, $F_{i, d}$ is the disturbance on the longitudinal dynamics. The disturbance force $F_{i, d}$ is the sum of the aerodynamic drag, rolling and road slope disturbances [27]: $F_{i, d}=F_{i, a}+F_{i, r}+F_{i, s}$. Drag disturbance depends on drag coefficient $C_{i, d}$, density of the air $\rho_{i}$, frontal area of the vehicle $A_{i}$, vehicle velocity $v_{i}: F_{i, d}=$ $0.5 C_{i, d} \rho_{i} A_{i} v_{i}^{2}$. Rolling resistance is a function of the vehicle mass, road surface coefficient $f_{i}$ and road slope angle $\alpha_{i}$ : $F_{i, r}=m_{i} g f_{i} \cos \left(\alpha_{i}\right)$. Finally, the disturbance is originated from the road slope: $F_{i, s}=m_{i} g \sin \alpha_{i}$.

\section{Simulation Results}

The efficiency of the proposed iterative control procedure for the determination of the passing order of autonomous vehicles at the intersection is demonstrated through two simulation examples. In the first scenario four autonomous 
vehicle arrives at the intersection whose ordering is determined to achieve the minimum traveling time, then the minimum energy of the vehicles. In the second example the passing order of five autonomous vehicle crossing the intersection is determined to reach the minimum traveling time of all vehicles.

\section{A. Energy and time-minimal control example}

In the first simulation example there are four autonomous vehicles at the intersection, three of them (Vehicle 1, 3 and 4) are turning left, while Vehicle 2 is heading straight. To determine the passing order of the vehicles to achieve minimum traveling time, the masses of the vehicles are considered to be homogenous. The velocity of the vehicles and the distances from the origin of the intersection are variant for all vehicles, see Figure 2.

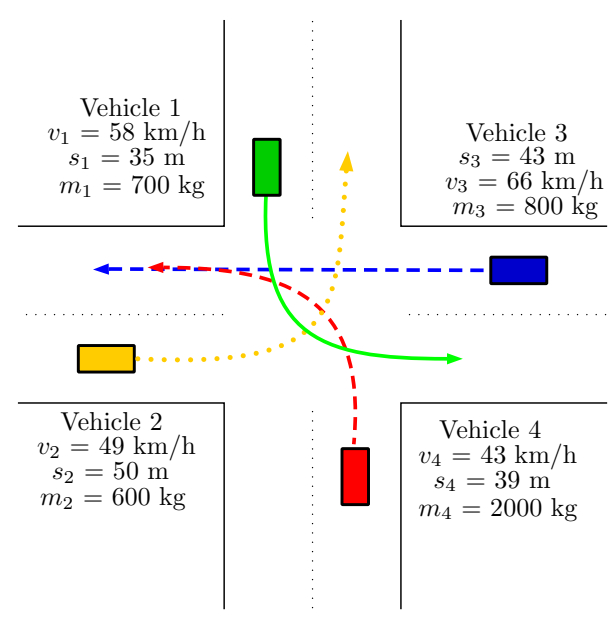

Fig. 2. Intersection scenario with four vehicles

The traveling time of all vehicles are calculated first, which serves as the basis to define ordering of the vehicles at the intersection and to evaluate the proposed time-optimal control algorithm. Therefore Vehicle 1 is the first vehicle turning left and leaving the intersection, then Vehicle 4 is the second. By significant deceleration Vehicle 3 allows Vehicle 2 to drive straight at the intersection, see Figure 3(a) showing the entry and exit time for each vehicles at the intersection conflict zone.

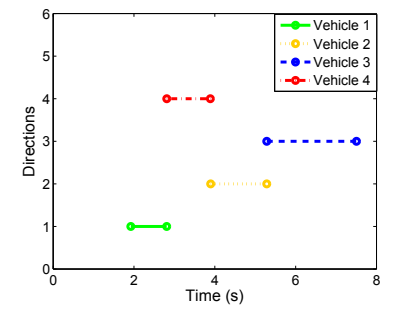

(a) Minimum time ordering

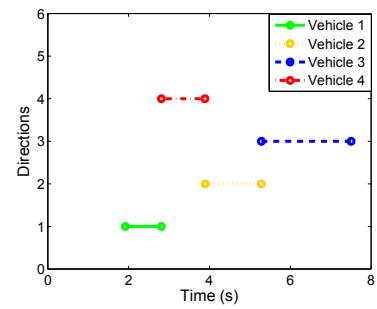

(b) Energy-optimal ordering
Fig. 3. Traveling time of vehicles in the conflict zone

To observe the energy-optimal case of the autonomous vehicles at the intersection, the masses of the vehicles are taken into consideration. There are no remarkable differences between the masses of Vehicle 1, 2 and $3\left(m_{1}=700, m_{2}=600\right.$ and $m_{3}=800 \mathrm{~kg}$ ), but the mass of Vehicle 4 is $m_{4}=2000 \mathrm{~kg}$. Since Vehicle 4 is close to the intersection and its mass is the biggest, it leaves the intersection first. Then the smaller Vehicle 2 with the relative high velocity $(49 \mathrm{~km} / \mathrm{h}$ ) for driving straight at the intersection is the second crossing vehicle followed by Vehicle 3. Opposite to the passing order of the case aimed at the achievement of the minimum traveling time, the last vehicle leaving the intersection is Vehicle 1, see Figure 3(b).

\section{B. Complex intersection example}

In this scenario there are five autonomous vehicles at the intersection whose positions and driving intentions are illustrated in Figure 4. Vehicle 1 and Vehicle 3 (Vehicles green and blue) are heading straight, while the other three vehicles (Vehicle 2, 4 and 5) are turning left. In this example the masses of the vehicles are considered to be homogenous, the distances from the origin of the intersection are $50 \mathrm{~m}$ in case of Vehicles 1, 2, 3 and 4. The distance of Vehicle 5 is $60 \mathrm{~m}$. Their initial velocities are $20 \mathrm{~km} / \mathrm{h}$, with the exception of Vehicle 4, whose velocity is $70 \mathrm{~km} / \mathrm{h}$. For safety reasons there are predefined speed limits for the vehicles: $v_{i, \max }$ is $50 \mathrm{~km} / \mathrm{h}$ for the vehicles heading straight, and $30 \mathrm{~km} / \mathrm{h}$ for vehicles turning left.

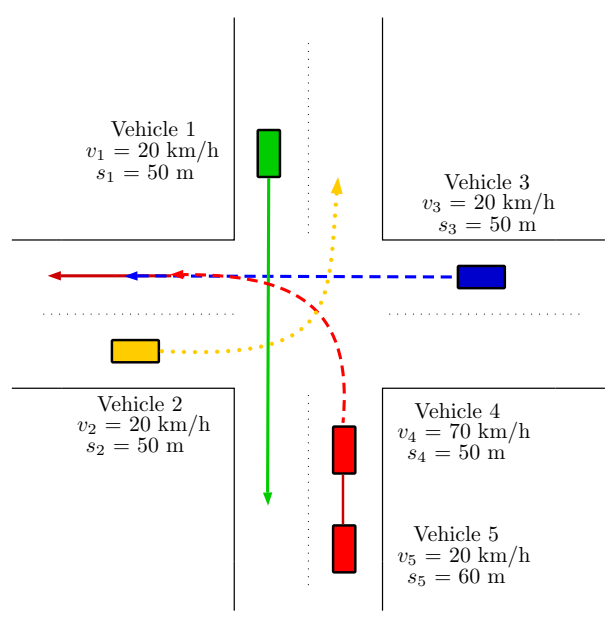

Fig. 4. Intersection scenario with five vehicles

In this intersection scenario there are several conflict situations between the autonomous vehicles considering their driving intentions. Taking into consideration the traveling time of vehicles, the first autonomous vehicle leaving the intersection behind is Vehicle 4 by the use of the proposed control method, see Figure 5(a). The skidding of Vehicle 4 is avoided by the deceleration to the maximum speed for turning left. At the time when Vehicle 4 leaves the conflict zone, Vehicle 5 already entered the control zone, thus ordering of the vehicles passing through the intersection is redefined by the recalculation of traveling time for each vehicles. For the purpose of achieving minimum traveling time, vehicles that are heading straight (Vehicle 3, then 
Vehicle 1) leave the intersection. Due to the control method, Vehicle 5, arriving behind Vehicle 4, crosses the intersection before Vehicle 2 (Figure 5(b)). All traveling times in the conflict zone and the crossing order are shown in Figure 5.

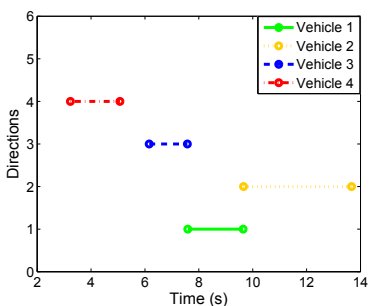

(a) First situation

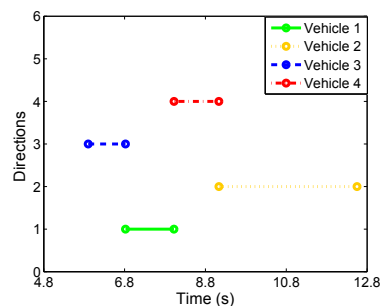

(b) Second situation
Fig. 5. Traveling time of vehicles in the conflict zone

The speed and acceleration values are illustrated in Figure 6(a) and Figure 6(b). It shows that the velocity of Vehicle 4 is quickly decreasing to achieve the maximum speed for turning left at the intersection. The velocities of Vehicles 1, 2 and 5 also are slowly decreasing while Vehicle 3 has increasing velocity until it reaches the maximum $50 \mathrm{~km} / \mathrm{h}$ speed allowed for vehicles driving straight.

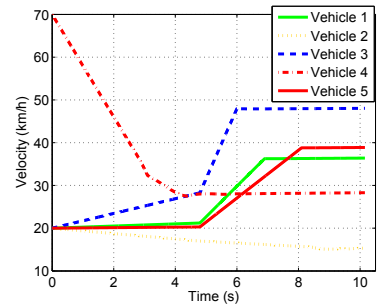

(a) Velocity

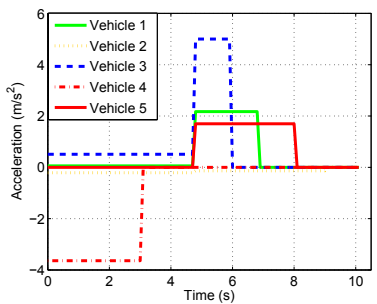

(b) Acceleration
Fig. 6. Velocities and accelerations of vehicles in the conflict zone

\section{CONCLUSION}

Ordering of vehicles at intersections without traffic lights or signs is affected by several factors. From the aspect of control design, the main purpose is to reduce traveling times while guaranteeing a collision-free passage. The paper proposed an iterative control procedure for autonomous vehicles crossing the intersection considering the minimum traveling time and energy consumption while avoiding collisions. The efficiency of the proposed control procedure has been demonstrated through various simulation examples.

\section{REFERENCES}

[1] L. Chen and C. Englund, "Cooperative intersection management: A survey," IEEE Trans. Intell. Transp. Sys., vol. 17, no. 2, pp. 570-586, 2016.

[2] K. Dresner and P. Stone, "A multiagent approach to autonomous intersection management." J. Artif. Intell. Res. (JAIR), vol. 31, pp. 591-656, 012008.

[3] I. Zohdy and H. Rakha, "Optimizing driverless vehicles at intersections," in 19th ITS World Congress, Vienna, Austria, October 2012.

[4] N. Murgovski, G. R. de Campos, and J. Sjöberg, "Convex modeling of conflict resolution at traffic intersections," in 54th IEEE Conference on Decision and Control, 2015, pp. 4708-4713.
[5] P. Dai, K. Liu, Q. Zhuge, E. H. Sha, V. C. S. Lee, and S. H. Son, "Quality-of-experience-oriented autonomous intersection control in vehicular networks," IEEE Trans. Intell. Transp. Sys., vol. 17, no. 7, pp. 1956-1967, 2016.

[6] L. Riegger, M. Carlander, N. Lidander, N. Murgovski, and J. Sjöberg, "Centralized mpc for autonomous intersection crossing," in 19th Int. Conf. on Intell. Transp. Sys., 2016, pp. 1372-1377.

[7] G. R. de Campos, P. Falcone, and J. Sjöberg, "Autonomous cooperative driving: A velocity-based negotiation approach for intersection crossing," in 16th Int. Conf. Intell. Transp. Sys. (ITSC), 2013, pp. $1456-1461$.

[8] R. Hult, M. Zanon, S. Gros, and P. Falcone, "Optimal coordination of automated vehicles at intersections: Theory and experiments," IEEE Transactions on Control Systems Technology, vol. 27, no. 6, pp. 25102525, Nov 2019.

[9] Y. Bichiou and H. A. Rakha, "Developing an optimal intersection control system for automated connected vehicles," IEEE Trans. Intell. Transp. Sys., vol. 20, no. 5, pp. 1908-1916, 2019.

[10] S. Liu, W. Zhang, X. Wu, S. Feng, X. Pei, and D. Yao, "A simulation system and speed guidance algorithms for intersection traffic control using connected vehicle technology," Tsinghua Science and Technology, vol. 24, no. 2, pp. 160-170, 2019.

[11] P. Gáspár and B. Németh, Predictive Cruise Control for Road Vehicles Using Road and Traffic Information. Springer International Publishing, 2018

[12] J. Lee and B. Park, "Development and evaluation of a cooperative vehicle intersection control algorithm under the connected vehicles environment," IEEE Trans. Intell. Transp. Sys., vol. 13, no. 1, pp. 8190, 2012.

[13] S. A. Fayazi, A. Vahidi, and A. Luckow, "Optimal scheduling of autonomous vehicle arrivals at intelligent intersections via milp," in American Control Conference, 2017, pp. 4920-4925.

[14] R. Hult, M. Zanon, S. Gros, and P. Falcone, "Energy-optimal coordination of autonomous vehicles at intersections," in 2018 European Control Conference (ECC), June 2018, pp. 602-607.

[15] S. Lefevre, D. Vasquez, and C. Laugier, "A survey on motion prediction and risk assessment for intelligent vehicles," Robomech Journal, vol. 1, 072014.

[16] O. Törô, T. Bécsi, S. Aradi, and P. Gáspár, "Sensitivity and performance evaluation of multiple-model state estimation algorithms for autonomous vehicle functions," J. Adv. Transp., pp. 1-13, 2019.

[17] N. J. Goodall, Real-Time Prediction of Vehicle Locations in a Connected Vehicle Environment. Virginia Cent. Transp. Inn. Res., Final Report, 2013.

[18] M. Casares, A. Almagambetov, and S. Velipasalar, "A robust algorithm for the detection of vehicle turn signals and brake lights," 9th Int. Conf. Adv. Vid. Sign.-Based Surv. (AVSS), pp. 386-391, 2012.

[19] A. Almagambetov, S. Velipasalar, and M. Casares, "Robust and computationally lightweight autonomous tracking of vehicle taillights and signal detection by embedded smart cameras," IEEE Trans. Ind. Electr., vol. 62, no. 6, pp. 3732-3741, 2015.

[20] Y. Shi, Y. Pan, Z. Zhang, Y. Li, and Y. Xiao, "A 5G-V2X based collaborative motion planning for autonomous industrial vehicles at road intersections," in Int. Conf. Sys, Man, Cyb. (SMC), 2018, pp. 3744-3748.

[21] A. Kokuti, A. Hussein, P. Marin, A. de la Escalera, and F. Garcia, "V2x communications architecture for off-road autonomous vehicles," in Int. Conf. Veh. Electr. Safe., 2017, pp. 69-74.

[22] Z. Shen, A. Mahmood, Y. Wang, and L. Wang, "Coordination of connected autonomous and human-operated vehicles at the intersection," Int. Conf. Adv. Intell. Mech., 2019.

[23] F. Gustafsson, "Slip-based tire-road friction estimation," Automatica, vol. 33, no. 6, pp. 1087-1099, 1997.

[24] K. Li, J. A. Misener, and K. Hedrick, "On-board road condition monitoring system using slip-based tyre-road friction estimation and wheel speed signal analysis," Automatica, vol. 221, no. 1, pp. 129-146, 2007

[25] L. Alvarez, J. Yi, R. Horowitz, and L. Olmos, "Dynamic friction model-based tire-road friction estimation and emergency braking control," J. Dynamic Systems Measurements and Control, vol. 127, no. 1, pp. 22-32, 2005

[26] Y. Bichiou and H. A. Rakha, "Real-time optimal intersection control system for automated/cooperative vehicles," Int. J. Transp. Sci. Techn., vol. 8, no. 1, pp. 1-12, 2019.

[27] R. Rajamani, Vehicle Dynamics and Control. Springer, 2005. 